INNOVATIONS IN PRIMARY CARE

\title{
Community Paramedic Mobile COVID-19 Unit Serving People Experiencing Homelessness
}

\author{
Zachary R. Stickler, NRP \\ Peter N. Carlson, MBA \\ Lucas Myers, NRP \\ Jill Ryan Scbultz, NRP
}

\author{
Teresa Swenson, NRP \\ Candice Darling, NRP \\ Chad Liedl, MS, RN \\ Rozalina G. McCoy, MS, \\ $M D$
}

Ann Fam Med 2021;19:562. https://doi.org/10.1370/afm.2709.

\section{THE INNOVATION}

In times of crisis, rapid deployment of clinical resources to underserved areas/populations is needed, particularly in the absence of an established health care infrastructure. The COVID-19 pandemic exposed profound gaps in care for people experiencing homelessness. ${ }^{1-3}$ A Mobile COVID-19 Unit was created with collaboration between Mayo Clinic Ambulance Service Community Paramedics and the Olmsted County Housing Stability Team in Rochester, Minnesota, to provide timely person-centered care to COVID-19-positive individuals experiencing homelessness.

\section{WHO \& WHERE}

The Mobile COVID-19 Unit was developed and staffed by 4 community paramedics (CPs), a community paramedic community health manager, and an internal medicine physician serving as the community paramedic medical director. Community paramedics are paramedics with advanced training in preventive care, chronic disease management, and social determinants of health. The Mobile COVID-19 Unit provided scheduled outreach to 3 facilities in Rochester that quarantined persons experiencing homelessness who had tested positive for COVID-19.

An estimated 459 people were experiencing homelessness in the area. ${ }^{4}$ People experiencing homelessness are at increased risk for COVID-19 complications due to high rates of comorbidities, prevalent smoking, and scarce caregiver support. ${ }^{5-7}$ Quarantined in a shelter, they also lack access to medical care outside of emergency medical services and departments (ED). There was urgent need to secure access to health care clinicians for evaluation of health status and monitoring of chronic health needs with the goal of preventing clinical deterioration.

Conflicts of interest: authors report none.

\section{Corresponding author}

Zachary R. Stickler

Mayo Clinic Ambulance Service

200 First Street SW

Rochester, MN 55905

stickler.zachary@mayo.edu

\begin{abstract}
HOW
Individuals were diagnosed with COVID-19 during communitywide testing organized by Olmsted County Public Health (OCPH) or at 2 area hospitals (Mayo Clinic and Olmsted Medical Center). After diagnosis, they were quarantined in 1 of 3 locations maintained by OCPH (2) and a local nonprofit organization (1). Care processes, workflows, and informatics solutions were developed within 2 weeks of the initial request for support from $\mathrm{OCPH}$.

In the first 2 weeks of Mobile COVID-19 Unit operations, 13 people were cared for. Community paramedics visited each site 6 days per week and patients were seen on each outreach day for the duration of their quarantine period. During each encounter, CPs accessed the patient's electronic health record (EHR), if available in our system, to review medical/social history and social determinants of health. CPs performed a comprehensive medical examination (including vital signs, oxygen saturation, cardiopulmonary, and extremity exams), medication review and confirmation of availability on site, and clinical risk assessment for severe COVID-19 disease. The community paramedic authored a note within the EHR, which was routed to the CP medical director when further review was needed. If patients needed chronically used medications, CPs facilitated their acquisition and, for medications usually administered in the clinical setting, administration. Support was available, if needed, through telehealth connection to the Mayo Clinic ED and the CP medical director.
\end{abstract}

\section{LEARNING}

The Mobile COVID-19 Unit was rapidly developed and deployed. It was successful in educating patients on selfmanagement, provision of timely medical care for COVID-19 and other acute and chronic health conditions, and building trust with this underserved community. The Unit also supported COVID-19 vaccination of unsheltered adults in conjunction with $\mathrm{OCPH}$. Success of this program was predicated on close communication between the CP team, Olmsted County Housing Stability Team, and shelter staff; versatility of CP skills spanning education, social support, and medical evaluation/management; and endorsement by community partners respected by the homeless community. The Unit is currently working toward expansion to all shelters and encampments, not limited to individuals with confirmed COVID-19 infection.

\footnotetext{
Supplemental material including author affiliations, funding support, acknowledgments, and references are available at https:II www.AnnFamMed.org/content/19/6/562/suppl/DC1/.
}

Key words: COVID-19, homeless, shelter, community paramedic, mobile health, public health; allied health personnel; homeless persons 